\title{
Design Evolution of the Zernike Phase Contrast Transmission Electron Microscope
}

S. Motoki*, T. Fukuda*, H. Suga*, Y. Okura*, R. Danev**, J. Brink*** and B.L. Armbruster***

* JEOL Ltd., 1-2 Musashino 3-Chome Akishima Tokyo 196-8558, Japan

**Okazaki Institute for Integrative Bioscience, National Institutes of Natural Sciences, Higashiyama 5-1, Myodaijicho, Okazaki 444-8787 Aichi, Japan

*** JEOL USA Inc., Peabody MA 01960

In the last decade the development of aberration correctors has improved the spatial resolution of scanning transmission electron microscopes and transmission electron microscopes (STEM/TEM) to better than $0.1 \mathrm{~nm}$. On the other hand, polymer researchers and biologists are eager to enhance sample contrast and detail in the mesoscopic scale $(1 \mathrm{~nm} \sim 100 \mathrm{~nm})$. To this end, the phase contrast imaging technique for TEM was proposed more than half a century ago, yet due to the absence of nanofabrication techniques, charging of phase plates and lack of precision control of the phase plate, this idea could only recently be realized [1,2]. Phase contrast TEM is now under intense investigation, and several experiments have shown the effectiveness of contrast enhancement at the mesoscopic scale. In this paper, we report the evolution and optimization of the Zernike phase contrast TEM.

The phase plate must be inserted in the backfocal plane to apply a phase shift of $\pi / 2$ to the scattered electrons versus the zero-order beam. Because high-resolution pole pieces generally have a narrow gap, the back focal plane of this configuration lies inside the lower pole piece. To make a phase plate for such an instrument, a dedicated transfer doublet lens system was built for the first and second generation column designs (Fig.1a, 1b) $[3,4]$. The design transfers the back focal plane to an optically equivalent position at the selected area aperture, thus affording better clearance for the phase plate holder. The third generation TEM, which adds the phase plate to a dedicated TEM column, includes the Zernike phase contrast pole piece (ZPCP) and a phase plate holder. Figure 1c shows the JEM-2200FS with the new pole piece and the phase plate holder installed. The new pole piece has a large gap that allows the phase plate to be inserted into the back focal plane of the objective lens. Figure 2 shows the relationship between focal length and point resolution and between focal length and cut-on frequency. As the figure shows, longer focal length creates a larger dispersion of the diffraction pattern, so that the cut-on frequency of the hole at the center of the phase plate can be minimized. To optimize observation in the mesoscopic scale with the phase plate, the pole piece was designed to have a focal length, spherical aberration coefficient and chromatic aberration coefficient of $5.2 \mathrm{~mm}, 4.2 \mathrm{~mm}$ and $3.9 \mathrm{~mm}$, respectively (see Fig.2).

In addition to the new pole piece, we are now developing an airlock for the phase plate holder and stage (Fig.3), which allows fast exchange of phase plates and more precise positioning of the phase plate. 


\section{References}

[1] R. Danev and K. Nagayama, Ultramicroscopy 88 (2001) 243.

[2] R. Danev et al., Ultramicroscopy 109 (2009) 312.

[3] F. Hosokawa et al., J. Elec. Microsc. 54 (2005) 317.

[4] S. Motoki et al., Microsc. Microanal. 11 (Suppl 2) (2005) 708.
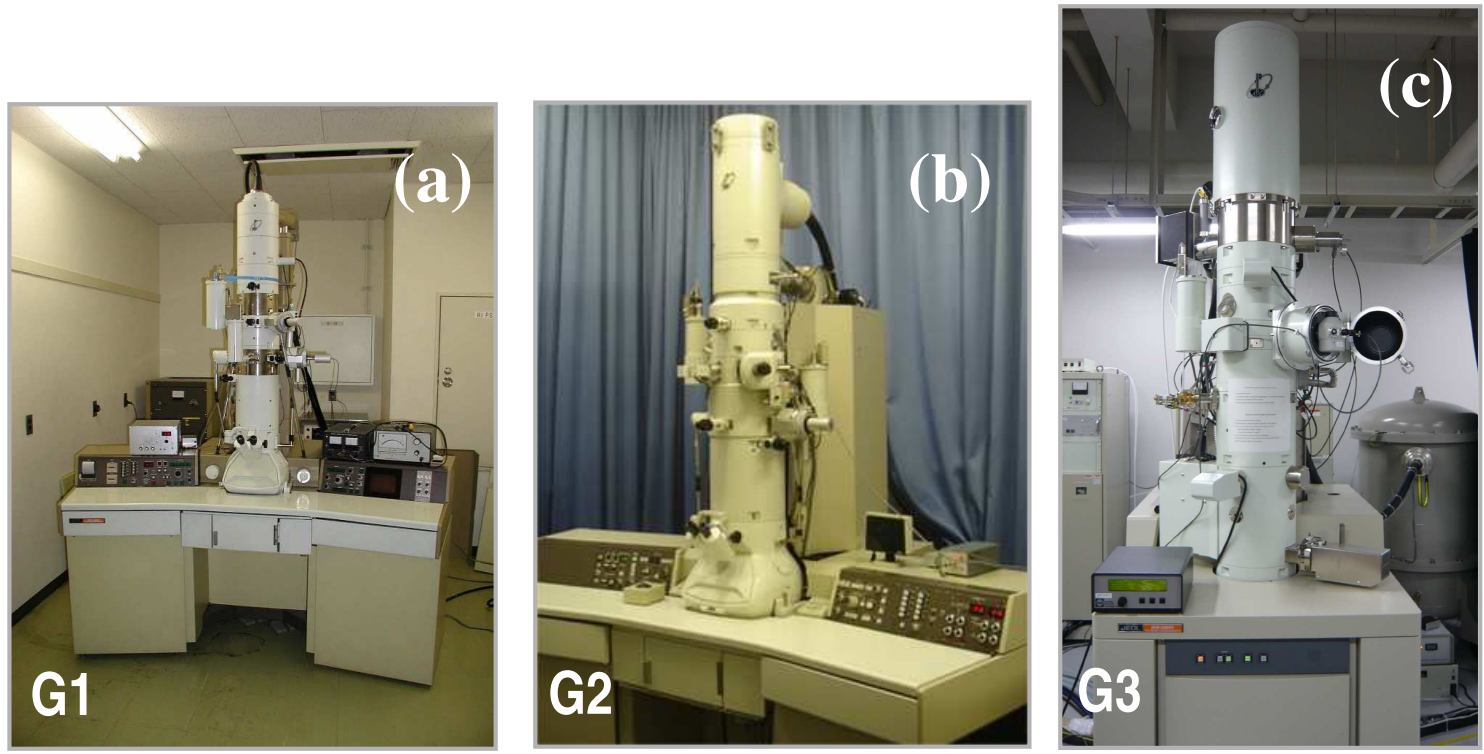

Figure 1. Appearance of the phase contrast TEMs at Okazaki Institute for Integrative Bioscience. (a) The first generation phase contrast TEM based on the JEM-1200EX. (b) The second-generation phase contrast TEM based on the JEM-2010. (c) The third generation phase contrast TEM based on JEM-2200FS.

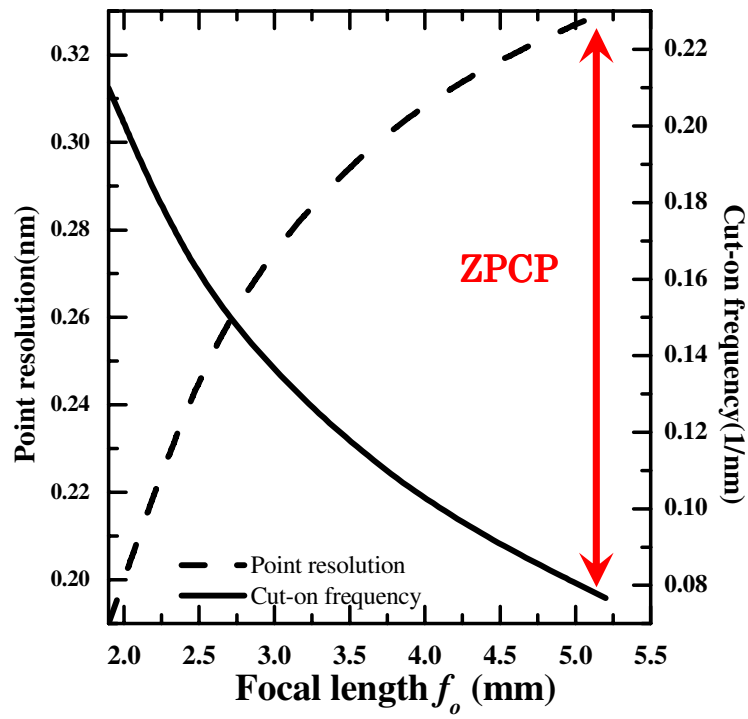

Figure 2. The general properties for point resolution and cut-on frequency depend on the focal length (fo).

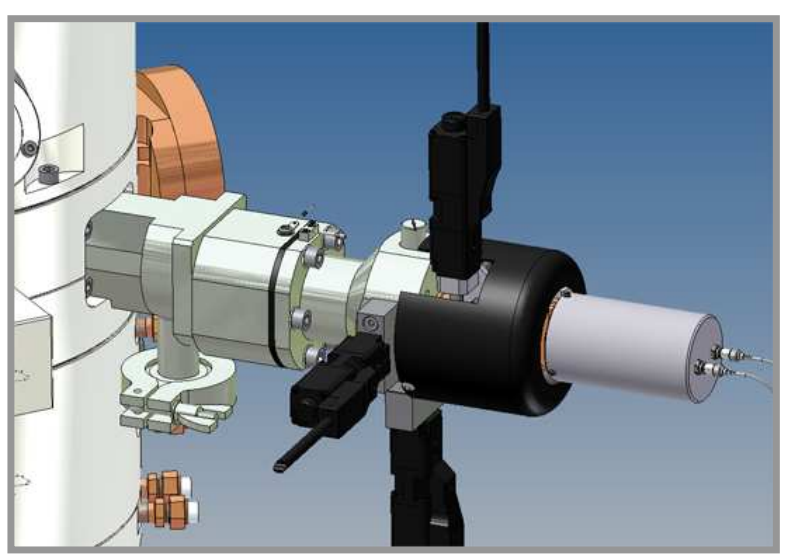

Figure 3. The air-lock type phase plate holder. The holder contains 2 stage piezo driver $(\mathrm{X}-\mathrm{Y})$ and 2 stage motor driver $(\mathrm{X}-\mathrm{Y})$ and ceramic heater for preventing the contamination and charging. 